\title{
Villes et migrants, du lieu-monde au lieu-passage
}

\author{
Lamia Missaoui et Alain Tarrius
}

\section{(2) OpenEdition}

\section{Journals}

Édition électronique

URL : https://journals.openedition.org/remi/2818

DOl : $10.4000 /$ remi.2818

ISSN : $1777-5418$

Éditeur

Université de Poitiers

\section{Édition imprimée}

Date de publication : 1 juillet 2006

Pagination : 43-65

ISBN : 2-911627-42-3

ISSN : 0765-0752

\section{Référence électronique}

Lamia Missaoui et Alain Tarrius, «Villes et migrants, du lieu-monde au lieu-passage », Revue européenne des migrations internationales [En ligne], vol. 22 - n² | 2006, mis en ligne le 01 juin 2009, consulté le 15 avril 2022. URL : http://journals.openedition.org/remi/2818; DOI : https://doi.org/ $10.4000 /$ remi.2818

Ce document a été généré automatiquement le 15 avril 2022.

(c) Université de Poitiers 


\title{
Villes et migrants, du lieu-monde au lieu-passage
}

\author{
Lamia Missaoui et Alain Tarrius
}

1 Une longue tradition de la sociologie et de l'anthropologie sociale suggère que les rapports entre l'étranger, le migrant, et la société qui l'accueille s'organisent autour de «l'entrée dans la ville »'. Lieu-monde, la ville propose les espaces résidentiels de regroupement, ceux de travail, de loisirs et de plaisirs, négocie les sociabilités des nouveaux venus avec les normes locales, dans la perspective d'une intégration de ces dernières. La ville serait une totalité capable, dans ses limites propres, de médiatiser le changement des appartenances, des identités, aujourd'hui et dans le temps des descendances générationnelles. Nos recherches nous permettent de douter de ces omniprésence et omnipuissance du « champ urbain » ainsi localisé intra muros, dans la négociation, voire la mutation des altérités.

2 La sociologie urbaine n'a pas échappé à l'influence des évolutionnismes du xix ${ }^{\text {ème }}$ siècle. C'est ainsi que Georg Simmel insiste sur l'autonomisation de l'individu en transit des milieux villageois, caractérisés par la répétition des rôles, aux entrelacs des sollicitations urbaines, des stimuli qui nécessitent choix et décisions (Rémy éd., 1995). Rapidement Robert Ezra Park reprendra ce point de vue à propos de l'étranger' ${ }^{2}$ de l'immigrant: du subi au choisi ${ }^{3}$, tel était le sens du parcours promis aux nouveaux arrivants étrangers, des campagnes ou de lointaines nations. La métropole dispensait sa pédagogie civilisatrice en éduquant l'immigrant aux conduites normées d'un monde totalisé dans ses propres limites: ainsi, l'entrée dans la ville consistait-elle, pour le nouveau venu, à trouver sa place dans la diversité des fonctions, des rythmes et des formes urbaines. L'étranger, disaient Park et Stonequist, est un voyageur bien fragile, livré à la souffrance de la découverte du monde dans le labyrinthe urbain : un « homme marginal », qui échappe aux siens sans être encore des nôtres. Appelé à négocier la diversité et les limites de ses désirs, de ses choix, en les situant dans les chassés-croisés des quartiers des riches et des pauvres, des nuits et des jours, du travail et de l'oisiveté, de la solitude et du lien, il devient, nolens volens, sujet démographique et moral des déséquilibres des formes urbaines : il est institué acteur des transformations urbaines. 
Inscrit dans les temps courts des multiples interactions, il est nécessairement acteur des temps longs de la ville.

Une dialectique opposant conservation et transformation produit de façon incessante l'homme urbain comme toujours nouveau: dès lors qu'une forme naît, une autre est déjà en œuvre pour la remplacer. Les clartés de l'officialité, du clairement dit et désigné, de l'espace en "pour et contre » de la conservation, de l'obnubilation sur les apparences des incessantes "crises", masquent les incertitudes, les périphéries, les marges, l'obscurité des processus de transformation qui n'apparaissent dans l'enceinte urbaine et en ses espaces publics que brièvement, sur le mode de l'incertitude. La dialectique conservation/transformation est mise en œuvre, en fait, par beaucoup plus de complexité qu'une opposition binaire. On peut en effet lire cette dialectique dans ses expressions les plus généralisées, par les débats politiques, les positions des médias,... bref, les discours clairement établis du pour et du contre. Des sociologies en restent d'ailleurs là dans l'approche des changements urbains, repérant en premier lieu les acteurs sociaux déterminés. Les apports les plus riches des écoles simméliennes nous apprennent que ce type d'analyses opère en trompe l'œil : il faut, disent-elles, intégrer un troisième élément à cette dialectique, qui, donc, passe d'une forme binaire à des formes ternaires: le clairement pour versus le clairement contre réalise un niveau indispensable de l'analyse, mais insuffisant; chacun de ces éléments entre en dialectique avec bien d'autres souvent occultés, amnésiés, invisibilisés de toutes façons par ceux qui ont le pouvoir de dire clairement le pour et le contre. Il s'agit des incertitudes, des désordres des marges, des processus de réinterprétation ou de renversement des valeurs ${ }^{4}$, des initiatives, des désirs des étrangers, de tout ce qui grouille de sens indicibles dans les mondes de l'altérité. La conservation a pour rôle ultime de masquer le plus longtemps possible la naissance, l'advenue de la prochaine domination du penser, de l'agir et du dire autrement. Cette conception hégélienne du changement, reprise par Simmel, est exemplarisée, dans les travaux de Park, par la notion obscure mais heuristique de "district moral $»^{5}$. En tel lieu de la ville se rencontrent, généralement en dehors des rythmes diurnes, des populations diverses par leurs origines et leurs conditions, rassemblées à partir de désirs individuels, et de mobilités dérogatoires de celles caractérisant les rythmes bien ordonnés de la fonctionnalité urbaine diurne. Remarquable pédagogie de la mobilité par la traversée de l'espace des autres, par le côtoiement, la banalisation et le partage d'une communauté des désirs, au-delà des spécificités culturelles, cultuelles et économiques : lieux de rencontres nocturnes pour consommer les produits prohibés, pour fréquenter des prostitué(e)s, des appartements d'échangisme (Welzer-Lang, 2005), des jardins publics (Gaissad, 2006) etc. rencontres diurnes pour engager des paris, jouer avec l'argent,... mutation de la valeur et des valeurs. Et voici peut-être pourquoi la «ville bouge ", change, et voici comment la fluidité de l'inscription nocturne des désirs de tous dans l'obscurité des rues, comme l'obscurité des processus mafieux de l'enrichissement, entre autres, s'associent à l'appétit des promoteurs immobiliers pour perturber un ordre urbain qui ne demandait qu'à reproduire l'illusoire clarté de son exposition, de l'invention de son efficience. Mais la clarté des expositions du pour et du contre n'est pas à même de saisir l'histoire incessante des transformations. Le clairement pour conserver les formes présentes est aussi illusoire que le clairement pour transformer ces formes dès lors qu'il s'agit de maîtriser le devenir de la ville. L'être, entré dans le monde par la ville, s'instruit de l'accumulation des singularités et des 
contradictions de la vie urbaine, et les amplifie, dans la ville, dans tous ses moments, et seulement là.

4 Cette séduisante conception de l'École de Chicago, toujours en vigueur dans bien des productions de chercheurs contemporains, est pourtant de moins en moins recevable telle quelle aujourd'hui. D'abord parce que la ville ne se saisit plus comme totalité, tant sa mise en forme dépend désormais d'espaces et de populations qui lui sont extérieurs, ensuite parce que les catégorisations de la norme et de la marge ne sont opposables que dans des franges de plus en plus restreintes d'une officialité chancelante marquée par la grande faiblesse des États à se dire, à s'exposer, à s'orienter, à incarner un projet social dans le champ de l'urbain.

Des migrants internationaux sont brusquement, en une vingtaine d'années, selon nos recherches (Tarrius, 1992; Tarrius, 2002; Missaoui et Tarrius, 1995), passés des soumissions au lieu-ville ${ }^{6}$ à celles des logiques commerciales apatrides des grandes firmes mondiales. L'exploitation n'est pas moins sordide, mais les modalités d'autonomisation du migrant sont autres. Savoir franchir les frontières nationales, celles des hiérarchies économiques et politiques, en les effaçant par la juxtaposition de réseaux commerciaux d'économie souterraine c'est substituer à l'impersonnalité des traités officiels, la chaleur des rapports interpersonnels étendus de nations en nations, autour de liens de confiance, d'engagements de parole, c'est contourner les traités internationaux, c'est atteindre des populations que les accords de richesse laissent dans la déshérence; c'est aussi ne plus dépendre des volontés des peuples et des nations traversés, mais demeurer dans la complémentarité de ses lieux d'origine, des siens, restés proches puisque uniques centralités; c'est encore relativiser à l'extrême les différences culturelles ou cultuelles, les xénophobies toujours liées aux exacerbations des identités locales, car faire commerce nécessite la connaissance et souvent le respect des autres partenaires, des autres espaces, des autres sociabilités. C'est, somme toute, réaliser le rêve civilisateur simmelien, mais moins dans l'enclos urbain, dans l'hostilité des réifications des quartiers de voisinages que dans le déploiement transnational des sociabilités. Nous avions, dans nos travaux, plutôt tendance à considérer que ces univers du parcours et de la sédentarité se déployaient de façon superposée, sans grandes interactions, à la façon de ce niveau masqué de la transformation. Des recherches que nous avons menées récemment ${ }^{7}$ nous permettent d'apercevoir des articulations entre grandes mobilisations transnationales et changements urbains. Ces topiques de la transformation n'apparaissent pas n'importe où, évidemment, mais tout près de frontières assignées, inventées comme limites économiques et culturelles radicales, idéologiques somme toute : nos travaux nous ont permis de les repérer dans les Sud de l'Europe, près du Maroc et de la Turquie. Plus précisément dans les villes de Sofia et d'Alicante. La trame des propositions de Simmel et de Park reste d'actualité comme mode privilégié de penser les processus de changement, mais la ville ne suffit plus à dire les sociabilités du monde contemporain : les « districts moraux » deviennent des zones criminogènes aussi vastes que l'Andalousie ou les espaces albanophones. Les mobilités intra urbaines ne sont plus que préparatoires à celles, désormais constituées en topiques civilisatrices, les "territoires circulatoires", qui traversent les nations (Tarrius, 2001). Changements d'échelles qui articulent au mieux les étrangers dans la ville avec leurs nombreuses origines : qui lui permettent désormais de s'affirmer d'ici et de là-bas, et de l'entre deux lieux. 
6 Nous désirons donc, dans les propos qui suivent, exposer des approches empiriques qui confortent la réalité de l'apparition de ces phénomènes nouveaux. Il est clair que notre critique rapide de Simmel et de l'Ecole de Chicago, de ses prolongements interactionnistes ne remet pas en question l'exigence du parcours de recherche empirique comme préalable à toute formulation de segments de théorisation. Conformément aux recommandations épistémologiques de Simmel nous n'essaierons pas, dans les exposés de terrain qui suivent, de démontrer le bien fondé de nos positions par l'exposé des seuls effets des présences de migrants transnationaux, mais nous tenterons plutôt de signaler l'apparition, même modeste, de ces effets dans la pluralité des facteurs de transformation de la ville.

\section{SOFIA : quand la gentrification " après le socialisme » se satisfait de l'étranger}

7 L'enquête menée à Sofia ${ }^{8}$ et sur les côtes bulgares de la Mer Noire, a été longue, car nous ne sommes pas là devant le cas courant des villes ex-socialistes en transition vers le capitalisme, où les transferts de propriété sont d'une part les marqueurs essentiels de la paupérisation accrue des populations, et d'autre part ceux de l'émergence d'une caste riche. Ici, d'emblée, les transformations urbaines ont été tributaires d'une double articulation à de vastes espaces extérieurs : au Moyen Orient par des migrants anciens, aux nations Ouest Européennes par des mafias puissantes. Trois populations témoins des transformations urbaines contemporaines exposent là, plus particulièrement, le processus de transition vers le capitalisme : les anciens et nouveaux migrants moyen orientaux, les Gitans et les populations paupérisées jusqu'à la misère, et enfin les milieux affairistes qui tirent de la rénovation urbaine et de divers trafics leur nouvelle richesse.

8 La population de Sofia a légèrement dépassé les 1300000 habitants. Cette ville, située à 500 mètres de hauteur, au pied d'une montagne mythique, la Vitocha, rassemble en son centre une zone d'architecture " socialiste » construite sur le modèle stalinien, dans les années cinquante, puis des rues bordées de petits immeubles et de maisons individuelles construites entre 1900 et 1930, par ci par là quelques maisons du XVIII ${ }^{\text {ème }}$, d'architecture austro-hongroise, et puis d'interminables étendues concentriques d'immeubles de quatre à six étages construits dans les années soixante-dix et quatrevingt.

\section{De la fraternité populaire à la main mise sur des secteurs commerciaux : les Syriens de Sofia}

La Bulgarie d'avant 1991 s'était solidarisée avec un «peuple frère » du Moyen Orient : la République de Syrie. L'important dispositif de formation bulgare recevait des élèves ingénieurs, des étudiants de hautes spécialités universitaires. Ceux-ci étaient issus pour partie des familles des dirigeants politiques et pour partie des familles de commerçants aisés et proches du pouvoir, des bazaris essentiellement. La bourgeoisie commerçante damascène préférait envoyer ses enfants à Sofia plutôt que dans la lointaine Moscou, non que les formations y soient meilleures, mais surtout parce que la proximité, via Istanbul, permettait de «faire passer » des marchandises absentes du marché bulgare, et recherchées par les habitants ayant quelque moyen. Un commerçant de vêtements, 
Syrien d'Alep, nous fit «faire le tour » de ses concitoyens installés dans le centre de Sofia, autour de l'ancien quartier des organes de pouvoir socialiste, quartier composé de vastes esplanades et d'immenses bâtiments, en cours de "gentrification mondiale ", tel un Palais du Parti réhabilité en Hilton : l'exposé que chacun me fit de sa trajectoire migratoire d'installation est d'un modèle unique :

Avant 91, on restait cinq ou six ans en Bulgarie, en partie pour les études, en partie pour perpétuer les commerces de bijoux et de vêtements qui existaient, entre Damas et Sofia, depuis le milieu des années soixante-dix. On n'était pas vraiment installés mais chacun savait qu'il pouvait acheter auprès des étudiants syriens. Après 1991, plusieurs centaines d'entre nous ont obtenu la naturalisation bulgare, et plusieurs centaines d'autres des autorisations de résidence à long terme ; alors nous avons installé des succursales de magasins de Damas ou d'Alep. Puis nous avons commercialisé, en plus des bijoux et des habits, des produits électroniques, en collaboration avec les Iraniens et les Afghans. Depuis cinq ans, notre quartier du centre reçoit des Égyptiens, des Palestiniens et des Tunisiens qui ouvrent des commerces de produits alimentaires et des petits restaurants à bas prix. Nous sommes très appréciés par les Bulgares qui sont de plus en plus attirés par ce quartier. Il n'y a pas de racisme contre nous, car nous ne volons les métiers d'aucun Bulgare, et on leur permet de faire de bonnes affaires par rapport aux commerces du centre ville.

10 Nous avons donc enquêté sur les migrations moyen-orientales en Bulgarie, entrant par Istanbul et les ports de Burgas et Varna ${ }^{9}$. Depuis 1997, après la grande crise qui vit le départ, et l'expulsion, de centaines de milliers de Bulgares turcophones vers la Turquie, un flux migratoire transnational s'est institué entre l'Afghanistan et le pourtour de la Mer Noire, via l'Iran, les Émirats (et surtout Dubaï), la Syrie, la Turquie, puis la Bulgarie, les retours s'effectuant généralement à partir du port bulgare de Burgas, vers la Georgie, via Odessa en Ukraine. Puis, par l'Azerbaïdjan, ces migrants retournent dans les villes et villages qu'ils ont quitté de douze à dix-huit mois auparavant. Nous sommes devant une de ces formes de migrations transnationales qui se développent de par le monde depuis les années quatre-vingt-dix. Ces migrants, des hommes presque exclusivement, travaillent par ci par là au cours de leur grande tournée, et, pour la plupart d'entre eux, servent de « porteurs » ou de passeurs à des commerçants installés à Dubaï ou en Syrie. Des Émirats, ce sont des produits électroniques de dernier cri, lecteurs MP3, micro-ordinateurs,..., qui transitent. Les «fourmis» afghanes ou iraniennes impliquées dans ces trafics agissent généralement sur commande de commerçants syriens de Sofia. Il s'agit de matériels détaxés et importés hors contingentement; leurs prix d'achat, par des Syriens installés à Dubaï sont inférieurs en moyenne de $65 \%$ aux prix pratiqués en Europe de l'ouest. Les reventes à Sofia les amènent de $-65 \%$ à $-40 \%$, ce qui représente un bénéfice net d'environ 3000 euros pour le convoyeur et de 2000 euros pour le commerçant dernier vendeur, sur un transport moyen de 20000 euros. De Damas et d'Alep, ce sont toujours les bijoux en or qui sont transportés: achetés $30 \%$ moins cher qu'à la revente en Bulgarie, cette transaction donne lieu à partage; une «fourmi » afghane des trafics peut raisonnablement espérer 4500 euros d'un passage. À Istanbul, des vêtements de cuir sont entassés dans des remorques. Ils sont cousus ou griffés, lorsqu'il s'agit de contre façons, dans le quartier à forte concentration d'ateliers afghans de confection près d'Autogar, la grande station de départ des autocars pour les Balkans. Une centaine de blousons de cuir revendus autour de 60 euros pièce, rapportent environ 1500 euros aux passeurs. Les gains effectués par des petits travaux tout le long du parcours, pourvoient aux frais de route et permettent d'envoyer fréquemment de petites sommes à la famille 
restée au pays. Enfin, après délestage à Sofia, où les commerçants syriens revendent sur place ou bien font circuler vers la Serbie, des invendus de voyages précédents sont acheminés, par un circuit maritime sur la Mer Noire, à partir de Burgas ou de Varna, vers la Roumanie, à Constanza, l'Ukraine, à Odessa, et enfin la Géorgie. Ce "complément de retour", rapporte environ 4000 euros. Dans une tournée d'une année, ces Afghans et Iraniens peuvent donc compter sur un gain d'environ 12 000 euros.

11 Sept semaines d'accompagnement de cars d'Istanbul à Varna ou Sofia, aux mois de juin et de juillet 2004, nous ont permis de situer très approximativement dans cette durée, le flux d'Afghans à 8000 personnes. Soit des autobus sont affrétés à Istanbul pour Sofia, soit des commerçants, essentiellement syriens, attendent les passeurs à Burgas, port proche de la frontière turque. Enfin, le retour d'une pratique locale bulgare présocialiste nous a été signalé : le gourbet ${ }^{10}$. Des habitants de villages se regroupent afin d'aller travailler (cueillettes, bâtiment,...) dans les pays balkaniques voisins. Nous avons, pour notre part, rencontré à Varna et à Burgas, et entre les deux ports, de tels groupes qui achetaient aux Afghans des marchandises destinées à la vente. Nous n'avons toutefois pu évaluer l'amplitude de leurs déplacements de revente.

Les Syriens bulgares sont donc au cœur de vastes transferts internationaux de marchandises. C'est probablement le rôle essentiel de ces flux de nouveaux migrants transnationaux, de plus en plus denses, d'acquérir et de développer une compétence circulatoire, qui se conforme étroitement aux projets de la mondialisation économique "sauvage ", parce qu'ultra libérale : faire parvenir par tous lieux et par tous temps aux moindres recoins solvables du globe, des marchandises dont les aléas des politiques nationales, désormais instituées comme locales par la toile mondiale de ces trafics incessants, pourraient les priver. Il est évidemment à première vue paradoxal de constater que ce sont des formes de contrebandes très en vigueur dans le monde précapitaliste ${ }^{11}$ qui se mettent au service de cette grande expansion du capitalisme....

13 La densification des populations "Arabes ", comme les désignent les démographes bulgares, au cœur de Sofia n'est donc pas un phénomène classique d'apparition de quartiers ethniques pauvres dans une ville en expansion: les 34000 Arabes, pour moitié en situation régulière et pour moitié sans autorisations, qu'a recensés l'ONG International Centre for Minoriy studies and Intercultural Relations de Sofia ${ }^{12}$, sont très généralement plus riches que les habitants bulgares de la ville, et représentent là l'irruption des savoir faire commerciaux moyen-orientaux, un vaste «Sud », du Caire à Islamabad. À la question sur l'absence de visibilité locale de cette richesse, le commerçant syrien que je rencontrai longuement me dit que le rôle des siens était de conforter leur position dans la ville avec discrétion ; l'étape d'ouverture de commerces de rue étant désormais bien entamée, il convenait de généraliser l'effort de réhabilitation des maisons anciennes qui forment leur quartier de résidence, plus que des rénovations souvent tapageuses et mal connotées dans Sofia.

Nous ne devons pas, me dit-il, être assimilés aux populations locales mafieuses, qui exhibent leurs richesses ostensiblement et provoquent une aversion profonde chez leurs concitoyens bulgares. Pour l'heure, ces mafieux jouent le rôle de miroirs aux alouettes et, conclut-il, quand l'Europe ou bien le peuple, selon un autre scénario politique possible, se débarrasseront d'eux, nous serons suffisamment solides, pour apparaître au grand jour comme ceux que nous sommes : de sincères concitoyens bulgares, ouvrant des perspectives économiques, donc politiques, inespérées voici 
peu, sur un Moyen-Orient, proche dans les périodes historiques fastes, et lointain

dans les temps de conflits et de misère ».

Lorsqu'on leur pose la question de la présence, à Sofia, de populations riches susceptibles d'agir sur les formes urbaines, nos collègues chercheurs bulgares répondent unanimement que ces personnes composent environ $15 \%$ de la population de la capitale, et que plus de la moitié d'entre elles tirent leurs revenus de pratiques mafieuses. Nous avons donc tenté de savoir plus précisément qui sont ces fameux $15 \%$. Cette proportion est en fait directement tirée de la population résidente dans des zones de la ville ou de ses environs, engagées depuis les années 1996 dans des rénovations hardies, souvent tapageuses, tels ce palace en forme de château fort, et les résidences qui l'entourent désignés par le sobriquet de "Beverly Hills", construits dans les quartiers collinaires de Dragalevtsi à Vladaya, au sud de Sofia, sur les premiers contreforts de la montagne Vitocha, dans la direction de la Macédoine voisine, là même où prospéraient jadis les seules villas de la nomenklatura. En fait, si nous retirons de la population de ces quartiers, les anciens propriétaires qui se sont maintenus là en louant des parties de leurs villas, leurs nouveaux locataires, les petits commerçants locaux ${ }^{13}$, les personnels de service des grandes villas, les nouveaux personnels politiques qui ont racheté des maisons modestes, les artisans (surtout du bâtiment) qui autoconstruisent des villas modestes, le chiffre de $15 \%$, usuellement donné, serait beaucoup plus près de $5 \%$. Les populations moyen-orientales sont absentes de ces quartiers. Là, se développent deux types de constructions : des rénovations d'anciennes villas, selon une échelle de la simple réhabilitation par peinture des façades et changement des fenêtres et des portes, jusqu'à des reconstructions hardies, gérées par des cabinets d'architecture, puis des constructions nouvelles, entourées de hauts murs de clôture surveillés par des caméras, et des vigiles habillés de noir, jusqu'au bonnet de laine, qui font au visiteur de larges signes d'avoir à décamper, avant de se saisir le leurs talkies-walkies au cas où il n'obtempère pas. Des chiens muselés, tenus en laisse, sont usuellement exhibés. Après une visite rapide de quelques sites, avec ma comparse résidente du quartier, visite rapidement achevée sous les injonctions des inévitables gardiens aux uniformes noirs, je retournai, costumé et seul, dans un de ces quartiers, qui me paraissait atteindre des sommets du mauvais goût hollywoodien. Là, l'hôtelpalace en forme de château fort de six étages, flanqué de tourelles d'angles et festonné de créneaux multicolores, trônait sur six regroupements de villas clôturées, un centre de loisirs aquatiques "relaxe, piscine, sauna ", des salles de musculation, et un centre médical, où les diverses spécialités, présentées en anglais sur un vaste panneau extérieur énuméraient les appartenances des praticiens à diverses associations internationales aux adresses anglaises, américaines et suisses. Je notai une adresse parisienne de société de néphrologie, que je ne pus identifier une fois revenu en France. Lors de cette visite, je parvins à entrer dans le hall de l'hôtel-château, où je demandai 
une chambre. Il me fut répondu que tout était complet : il n'y avait aucune voiture sur le parking, toutes les clefs des chambres pendaient sur un présentoir et les rideaux de toutes les fenêtres étaient clos. Puis je me dirigeai en boitant vers le cabinet médical, ayant appris par le grand panneau la présence d'un ostéopathe correspondant d'une Académie londonienne. Une réceptionniste me dit qu'il s'agissait d'une «fondation privée internationale » et que je ne pouvais consulter que sur rendez-vous. Lorsque j'arguai d'une urgence, elle me proposa de téléphoner à une ambulance pour me conduire dans un hôpital de Sofia. Je longeai l'établissement de "relaxe, piscine, sauna ", qui, lui, résonnait des cris de baigneurs, et entrai dans un de ces espaces clôturés qui agrégeait sept grandes villas. Deux personnes âgées, revêtues d'un uniforme gris, me firent signe de rebrousser chemin. Je montrai une villa en bout de rue et continuai mon chemin : une voiture arriva alors très rapidement de je ne sais où, et deux « vigiles vêtus de noir " me proposèrent très poliment - «it's enough for today " me dirent-ils en riant - de m'asseoir à l'arrière puis me raccompagnèrent sur la petite place de Dragalevtsi où m'attendaient, dans le minuscule estaminet, Svetla et trois autres personnes. Il s'agissait d'employés d'entretien des «établissements » que j'avais tenté de visiter. Chacun me dit qu'il n'y avait jamais de client dans l'hôtel et pas plus de médecins dans le centre médical, mais que, par contre, le ballet des grandes 4 x 4 noires BMW, Mercedes et Porsche, ces dernières étant les plus nombreuses, était incessant, avec, toujours, à bord, derrière des vitres noires, les inévitables vigiles. Il était difficile de faire mieux en matière de mise en scène de séries $B$ hollywoodiennes. Mes interlocuteurs insistèrent sur la morgue de ces " nouveaux riches ", sur le fait que l'on ne connaissait pas les origines de leurs fortunes, sinon, pour quelques uns l'expropriation, dans Sofia, de multiples familles. Par ailleurs ils me signalèrent que ces personnes, malgré elles, étaient en train de provoquer la venue de milliers de visiteurs, pour voir « le spectacle » depuis l'axe routier principal, et qui consommaient sur place. Les estaminets comme celui de Svetla étaient en effet nombreux, et, vers trois heures de l'après-midi, ce samedi là, plusieurs centaines de personnes débarquèrent des autobus des lignes 64 et 98, donnant à la place l'allure d'une kermesse. Les langues se déliaient pour dénoncer ces abus de richesse, et quelques uns allaient en excursion vers les portails des enclos surmontés des créneaux du château-hôtel, avant de se faire copieusement injurier par les vigiles regroupés derrière une barrière de chiens. Parfois une voiture luxueuse passait et ralentissait sous les quolibets de la foule; les vitres noires étaient remontées, et il arriva qu'un occupant d'une somptueuse voiture montre sa tête pour cracher en direction de la foule. Les anciens membres de la nomenklatura, ou leurs enfants, servaient sandwiches et friandises cuisinés à la maison et enroulées dans du papier journal en exacerbant, par leur colère quotidienne, l'indignation collective. Les propos échangés entre toutes ces personnes, qui prenaient leurs voisins à témoin, étaient indignés : « voilà où l'on en est ! C'est ça la liberté promise! " «C'est pire qu'avant " disaient d'autres, attirant la réponse des ex-apparatchiks «jamais nous n'avons craché sur le peuple » phrases clefs échangées sur cette place « du spectacle de la gentrification ». Phrases entendues très souvent ailleurs dans Sofia, amplifiées par la rumeur. 


\section{Gentrification et paupérisation liées à l'émergence des « nouveaux riches »}

L'allusion qui m'avait été faite, par les trois compagnons de Svetla, et par une collègue universitaire, aux spoliations d'appartements par des « mafieux » correspondait bien à la réalité. A partir de 1993, et surtout dans les quartiers sud proches de Touka, où on abandonne le tramway centre urbain $n^{\circ} 14$, pour prendre les bus 64 et 98 en direction de Dragalevtsi, des commerces «toucrédit» s'installèrent au bas d'immeubles construits dans les années soixante-dix et quatre-vingt dont les logements appartenaient à d'anciens fonctionnaires moyens, souvent demeurés en poste, mais aux salaires désormais miséreux de 80 à 120 euros par mois. Ils proposèrent aux habitants propriétaires d'appartements des formules de « crédit cumulatif à long terme ", c'est-àdire d'ardoises permettant d'acheter, quand nécessaire, utile, ou agréable, de l'alimentation, des vêtements, des appareils électroménagers, audio-visuels, ou d'autres applications électroniques. Au bout de deux ou trois ans la note était présentée aux familles qui, ne pouvant la régler, étaient expulsées avec un pécule leur permettant à peine d'aller se loger dans de vieux appartements délabrés du centre ville. Lorsque l'immeuble entier est ainsi accaparé par le commerce " toucrédit », une réhabilitation s'en suit, attirant des familles aux revenus de 250 à 400 euros, susceptibles d'évoluer rapidement après l'intégration européenne, auxquelles sont proposées des formules de « location achat » directement garanties par les gérants du commerce «toucrédit » de rez de chaussée qui, dès lors, migre vers un autre immeuble. Ces deux dernières années, les prix de l'immobilier ont été multipliés par cinq, sous l'influence conjuguée des transactions que nous venons de signaler, et de l'arrivée d'investisseurs britanniques, qui créent des petits ghettos de réhabilitations, dans quelques quartiers de Sofia, ou encore dans des villages ruraux, déstructurant les modes d'exploitation agricole antérieurs. Les banques bulgares, actuellement, ne prêtent pas pour de tels achats. Des petits commerces de proximité, des cafés, des coiffeurs, etc., sans lien avec les précédents "toucrédit", s'installent dans les rez-de-chaussée, et tout ce monde là attend sa promotion au statut de "nouvelle classe moyenne », adoptant par avance les comportements supposés de celle-ci. Mais cette population est terriblement fragilisée par la stagnation de son pouvoir d'achat face à l'augmentation du foncier : la plupart de ces habitants ont contracté des emprunts auprès de leurs interlocuteurs immobiliers mafieux, avec des clauses de réajustement de la dette en fonction de l'évolution du coût de la pierre... Une " gentrification du pauvre » nourrit l'autre, celle du riche, en somme.

Les manipulations autour du foncier ne sont pas les seules sources d'enrichissement des populations mafieuses. La Bulgarie est une voie privilégiée des trafics d'héroïne afghane ou turque (c'est souvent la même morphine base afghane, qui est transformée en Turquie). Les «fourmis» afghanes qui ne pratiquent pas les détours par le Golfe, pour s'en tenir à des trafics de produits d'usages illicites, passent directement par le nord de l'Iran, ou l'Azerbaïdjan, puis par la Turquie, notamment embarquent à Trébizonde pour Burgas, pour les premiers, la Géorgie et la Mer Noire pour les seconds, et déferlent en Bulgarie. Les turcophones présents dans le Sud de la Bulgarie, plus ou moins assistés par des Roms ${ }^{14}$ musulmans, relayent les Afghans pour les passages en Macédoine, puis dans la région "sans Etat" de la "grande Albanie », à l'Ouest du Kosovo, afin d'atteindre le Monténégro et d'y embarquer, dans les petits ports côtiers 
de l'Adriatique, les psychotropes à destination de l'Italie. Une vaste zone criminogène s'étend à l'Ouest de Sofia et de la Bulgarie.

Les Roms concernés par ces commerces sont en réalité peu nombreux : beaucoup plus, comme ailleurs en Europe, autoconsomment l'hérö̈ne de très mauvaise qualité qu'ils transportent, provoquant l'intervention d'ONG, telle Médecins du Monde.

Deux regroupements de Roms existent depuis plus de trente ans dans Sofia, l'un à l'Ouest, le long de l'avenue Alexandrov, regroupe des petites maisons: nostalgiques, leurs habitants montrent les balais et les vêtements qu'ils enfilaient voici encore quinze années, quand un emploi au moins était attribué à chaque famille, pour l'entretien des rues. L'autre à l'Est, près de la gare, est formé d'immeubles dans un état sordide aujourd'hui. Maladies et misère ont rapidement dégradé l'aspect même de cette population : habits déchirés des enfants, qui mendient, mères assises en rond sur le sol humide des cours, véhicules rouillés, amoncellements de déchets au rez-de-chaussée, au droit des fenêtres. La complémentarité économique des Roms, en ville comme à la campagne, dans les coopératives où ils occupaient de nombreux emplois, est morte depuis le changement de régime. Et, si quelques rares intérieurs cossus, dans ces quartiers, témoignent de l'implication de certains dans les trafics, la misère est généralisée. À Podgorica, au Monténégro, où nous avons vu arriver des camions de Bulgarie, qui facilitent la circulation des psychotropes dans ces zones «sans État » et effectuent du troc avec les habitants apparemment délaissés de ces zones. Nous n'avons noté la présence d'aucun conducteur ou accompagnateur Rom ; par contre les camions appartenaient à des compagnies gérées par des Bulgares présents sur les coteaux gentrifiés de Dragalevtsi.

En somme, l'enrichissement inconsidéré de quelques uns, acteurs d'une gentrification outrancièrement provocatrice, et d'une tentative de gentrification, via les réhabilitations d'immeubles, d'une population fragile et manipulée, se paie par la déshérence des plus pauvres, c'est-à-dire des plus nombreux, par l'approfondissement des stigmates et de la xénophobie qui les concernent.

\section{Le « sort du commun »}

21 Nous venons d'envisager la situation d'environ $20 \%$ de la population de Sofia. Pour les habitants partageant le sort commun, les salaires, quand il y a du travail (plus de $27 \%$ de chômeurs) ne dépassent guère cent euros par mois. Une visite des grands marchés ouverts, et des commerces de rues, nous a permis de noter les coûts des produits les plus utilisés pour l'alimentation. Un kilogramme d'oignons, de tomates, de fruits, ou un litre de vin : de 0,4 à 0,6 euro, navets, pommes de terre, 0,3 euro, haricots secs, 0,8 euro. Ventrèche fumée, 2,5 à 3 euros le kg. Bas morceaux de bœuf, 5 euros kg. Porc frais, 2 à 2,5 euros. Poisson sec, 3 euros le kg, et frais, 6 euros. Les fruits et les légumes s'achètent à l'unité : une mandarine par ci, trois pommes de terre par là... Les loyers, dans les immeubles construits dans les années soixante-dix et quatre-vingt, qui recouvrent l'essentiel de cette ville reconstruite dans l'après Deuxième Guerre mondiale : 60 euros un deux pièces, 80 un trois pièces. Les aides sociales sont minimes : environ 20 euros par enfant. Voici donc à résoudre une équation, dont le résultat nous dit qu'il est impossible pour une famille de vivre actuellement à Sofia. Des familles se regroupent dans un seul petit appartement, d'autres quittent la ville pour des villages; toutes entretiennent des liens très étroits avec leurs parents ou amis ruraux : les entassements 
de pots de confitures, de légumes, de confits, sont impressionnants. Les plafonds de cuisines supportent des dizaines de ventrèches ou de saucissons. Chacun, qui peut louer un lit ou une pièce accepte un entassement ailleurs. Dans un square central, entre le Ministère des Affaires Économiques Internationales et une grande Eglise Orthodoxe Serbe, nous avons compté 127 personnes sans domicile, un vendredi après-midi. Ce qui semblait être une manifestation ou un attroupement en vue, par exemple, d'une distribution de nourriture ou de vêtements, n'obéissait en fait à aucune de ces opportunités. Un prêtre, à l'entrée de l'église orthodoxe, me dit que là, ces personnes ne craignaient pas la police car «en cas d'arrivée des camions qui les ramassent et les amènent en banlieue, ils entrent tous dans mon église où ils sont intouchables".

Les nostalgiques de la période socialiste sont désormais légions, et se comptent non seulement parmi les populations qui vivent de ces petits revenus mensuels, mais aussi parmi les professions de l'enseignement (assistant d'université, 150 euros, professeur, 350 euros, instituteur, 180 euros). Le spectacle des rues, la solvabilité des chalands n'étant pas au rendez-vous, est triste, dès que l'on dépasse les quelques avenues autour de l'hyper centre : très peu de commerces, produits proposés obsolètes. Le « quartier arabe » est très fréquenté non seulement par tous ceux qui cherchent des produits bon marché et neufs, mais aussi par des promeneurs, des familles qui flânent dans ces rues propres et laborieuses.

23 Ainsi donc va la "transition vers le capitalisme ", thème idéologique inlassable de la demande faite par le pouvoir d'attendre encore un peu : environ $45 \%$ de la population urbaine dans une pauvreté profonde, $20 \%$ dans la misère, $15 \%$, les étrangers, autoconstruisant leur développement, $12 \%$ vivant « dignement » avec des salaires de 400 à 800 euros, et environ $8 \%$ exposant une richesse inouïe.

Dans un contexte historique très différent, l'évolution actuelle de la ville d'Alicante laisse apparaître, elle aussi, l'influence des migrants transnationaux; ce fait est particulièrement intéressant, puisqu'il nous dit que le «partenariat» de ces nouvelles formes migratoires dans les transformations locales n'est pas tributaire de leurs spécificités historiques. C'est sur les mêmes bases d'une description de l'ensemble des tendances du changement, que nous décrirons Alicante, après Sofia.

\section{ALICANTE : réhabilitations et gentrification dans une ville « mondialisée » 15}

25 Alicante, à l'image du grand port voisin de Valencia, à $170 \mathrm{~km}$ au nord, a historiquement joué le rôle de porte ouverte de l'Espagne, et plus précisément de Madrid, sur la Méditerranée. Le port de Valencia s'est plutôt spécialisé dans les grands flux de marchandises, et a beaucoup "tiré » le développement de sa ville dès lors que les implantations industrielles se multiplièrent près de ses môles, au xix ${ }^{\text {ème }}$ siècle; le trafic des produits agricoles, durant le $\mathrm{xx}^{\mathrm{ème}}$, accrut encore cette puissance, sans supplanter le rôle des industries. Quant à Alicante, son port était plutôt, comme il l'est encore, réservé aux voyageurs ainsi qu'aux produits manufacturés à Madrid. Ceux-ci sont présentés dans des boutiques près des quais aux commerçants venus du pourtour méditerranéen, qui peuvent les acheter ou passer des commandes par quantités plus importantes. Toutes deux dans l'aire catalane, Valencia et Alicante se différencient historiquement par leurs populations contrastées: milieux ouvriers de forte densité 
pour la première, populations d'origines espagnoles et étrangères variées, autour des métiers du commerce, pour la seconde. Valencia comptait 777000 habitants en 2000 et 747000 en 2005, et Alicante respectivement 275000 et 267000 . Toutefois, alors que la première se présente comme une ville compacte séparée de son port, Alicante, dont la ville basse donne directement sur le port, est au cœur d'une conurbation de villes: Benidorm à 40 kilomètres au nord, Santa Pola à 17 au sud, Elxe à 22 et Crévillente à 27 kilomètres à l'ouest. Les échanges sont constants et de forte intensité entre ces villes, de telle sorte que le dimensionnement réel des services et commerces d'Alicante concerne plus de 450000 habitants. La perte de 8000 habitants à Alicante, entre 2000 et 2005 a été largement compensée par une augmentation de la population dans les cités en conurbation.

\section{Étages territoriaux et populations spécifiques à Alicante} Petites maisons aux façades peintes à la chaux, aux portes bleues et aux balcons recouverts de carrelages, dans une végétation de minuscules jardins méditerranéens (figuiers, grenadiers, bougainvillées, agrumes, treilles,...) constituent le " vieux village » d'Alicante, dénommé "casq antic », désignation fréquente, en Espagne, des centres urbains historiques. La partie nord du port, était, jusque dans les années soixante entièrement réservée aux pêcheurs inscrits maritimes, petits métiers et pêches plus importantes : les maisons du casq antic constituaient l'habitat de ces marins. Cette unité entre quartier, vieux port et population, laissa place, à partir des années soixante-dix, à une autre unité « de remplacement » : des Britanniques achetèrent $85 \%$ des maisons de pêcheurs, mouillèrent leurs bateaux de plaisance dans le vieux port, et la municipalité créa, à partir des années quatre-vingt, un vaste ensemble immobilier et hôtelier de luxe sur la jetée nord. Bars, restaurants de poissons, boîtes de nuit, se pressent là, le long des appontements réhabilités, séparés du casq antic par une barrière d'immeubles 1930, qui hébergent des administrations et protègent les résidents britanniques des bruits nocturnes du port.

Cette " enclave » urbaine et portuaire est largement ouverte sur la partie maritime de la conurbation : en effet, ce dispositif nocturne touristique, enrichi depuis peu par un grand casino sur la partie sud du vieux port (un tunnel sous l'entrée de la rade joint les deux quais), draine tous les soirs, de 17 heures environ à quatre heures du matin, des processions de bateaux de luxe venus de Benidorm, de Santa Pola et de diverses petites stations balnéaires. Les valeurs du mètre carré résidentiel sont exorbitantes par rapport aux valeurs des autres quartiers d'Alicante : en juillet 2005 une maison du casq antic, de $90 \mathrm{~m}^{2}$ environ, avec un jardin de $20 \mathrm{~m}^{2}$ a été vendue près d'un million d'euros, alors qu'à 300 mètres de là, ans la vieille ville, des logements de 100 à $120 \mathrm{~m}^{2}$ en très bon état étaient mis en vente entre 108000 à 140000 euros. La famille espagnole modeste, descendante directe de pêcheurs, contactée quelques jours avant la transaction nous dit sa haine pour les nouveaux venus : 
Partout en Espagne il faut partir des vieux quartiers sur la mer. [...] On ne pouvait plus vivre avec ces Anglais ; ils nous imitent mais ça sonne creux ; [...] maintenant tu sors et tu croises une anglaise avec des mantilles, alors tu n'en mets plus [...], et tout est comme ça, tu te sens complètement ridicule dans tout ce que tu fais, que tu manges, que tu regardes, que tu dis. [...] On est presque les derniers à tenir, et on s'en va, en Espagne, là-bas, de l'autre côté de la Rambla Nunez, à huit cent mètres d'ici, en Espagne.

Exotisation insupportable pour les uns et création d'une culture spécifique pour les autres... qui les rattache symboliquement aux anciennes populations de bord de mer, et de fait aux populations riches internationales présentes d'Almeria à Cadaquès sur la bordure méditerranéenne. Cette partie d'Alicante, extraite, en quelque sorte, de la ville, est en conurbation avec tous les autres lieux distingués des côtes européennes de Méditerranée, et, si l'on en croit la fréquentation de son pôle portuaire touristique et l'immatriculation des bateaux de plaisance, avec bien d'autres contrées habitées ou visitées par ce qu'il est convenu d'appeler la «jet society»: Saoudiens et Émirates, Russes, et franges excessivement riches de nombreuses nations pauvres, sont présents là, sur les quais nord du port d'Alicante, introduits en quelque sorte par la petite population anglaise du casq antic. Il y a là gentrification, certes, et mixité d'origines, mais en aucun cas mixité avec le reste de la ville. Le casq antic est devenu un lieu de centralité de nombreuses populations nordiques qui désormais travaillent à distance de leurs entreprises, via internet, sur cette côte espagnole, ou encore partagent entre nord et sud leur temps de travail puis de vie familiale.

\section{La « ville basse »}

Voisine du casq antic et longeant le port de plaisance vers le sud, la vieille ville basse maritime regroupait jusqu'au début des années quatre-vingt une lisière d'hôtels, sur le modèle des quartiers hôteliers de front de mer de Cannes, de Nice et d'autres villes de la Riviera italienne. Une allée magnifiquement arborée, l'Explanada d'España, les sépare des quais. Ces hôtels, supplantés par ceux construits sur le quai nord, exposent des façades grisâtres et abritent en rez-de-chaussée des restaurants bon marché. Derrière ces immeuble, et sur une profondeur d'environ trois cents mètres, la ville étrangère des "bazars et pensions». C'est là que sont apparus, durant les années quatre-vingt-dix, en simultanéité avec les réhabilitations et les constructions du casq antic et du quai nord, des dizaines de commerces tenus par des Algériens et des Marocains, et des locations, du type "pension " pour les résidents de ces deux nations de passage. C'est là encore que résident, dans des locations bon marché, des Maghrébins qui travaillent dans cette même ville basse, dans la restauration, l'hôtellerie, et le commerce. Mais ce lieu n'a rien à voir avec les quartiers maghrébins pauvres qui peuplent nos banlieues ou certains centres villes en déshérence: les associations de commerçants marocains et algériens ont fédéré des ressources de leurs membres afin d'acheter et de rénover de nombreux immeubles de ce quartier et personne, qui réside là, n'est au chômage ; il s'agit d'un quartier « de la réussite », nous disent plusieurs commerçants et les responsables municipaux de l'urbanisme.

Ce quartier d'Alicante est lui aussi ouvert sur la conurbation et sur l'internationalité : Crévillente, ville de plus de 30000 habitants, à l'ouest d'Alicante et d'Elche, étape sur l'autoroute du pourtour méditerranéen, entre l'Italie, Marseille et le Maroc, concentre plus de cinquante commerces de tapis et d'appareils électroniques, que "chargent " quotidiennement les véhicules de milliers de «fourmis», petits migrants qui 
pratiquent le commerce international vers le Maroc et l'Algérie. Au grand quai est du port tout proche, véritable frontière maritime avec Oran et Melilla, des milliers d'Algériens et de Marocains, venus d'Europe et bien sûr d'Espagne, embarquent sur des ferrys. Ainsi, rattaché aux incessants flux commerciaux terrestres de migrants commerçants résidant dans les nations européennes par l'autoroute et par le port, ce petit quartier d'Alicante peut rivaliser, en matière d'amplitude et d'intensité des mobilités, avec son riche voisin du casq antic. Mais les populations des deux quartiers n'ont rien à négocier entre elles.

Dans quelques appartements non réhabilités logent des personnes très pauvres, sur le mode de locations collectives ou de squats. Là, comme dans bien d'autres villes européennes, et quelle que soit la tolérance manifestée par les autorités vis-à-vis des commerçants maghrébins, le stigmate de l'« Arabe » se mêle bien à celui du pauvre. Par contre, ces Maghrébins entretiennent des liens privilégiés, nous le verrons plus avant, avec une autre population spécifique alicantine: les Pied noirs rapatriés d'Algérie au début des années soixante. On peut donc observer, dans ces zones centrales de la ville, la juxtaposition de deux mondes, deux parts de la cité, abstraits de l'agglomération d'Alicante. Dans le quartier des commerçants Maghrébins, la municipalité a entrepris des réhabilitations de rues, trottoirs et façades, sans que l'on sache trop s'il s'agit de ne pas trop effaroucher les voisins riches du casq antic et du quai nord, ou d'accompagner le dynamisme commercial maghrébin par quelques aménagements. Un responsable de l'urbanisme, à la municipalité, nous affirme simplement que, dès lors qu'émerge une population étrangère à Alicante, la tradition consiste à l'installer au mieux, dans l'intérêt de tous les artisans et commerçants de la ville. Il est toutefois difficile de s'en tenir à cette définition : l'exemple de la misère des Gitans, cantonnés dans un quartier nord de la ville dans des cités insalubres, puis chassés pour on ne sait où, à l'occasion d'extensions immobilières, ou des Marocains pauvres, récemment arrivés pour travailler dans les services et l'agriculture, dans des friches portuaires au sud, est là pour tempérer cette déclaration très conforme à l'idée locale du cosmopolitisme alicantin. En fait il semble bien que ces deux "réussites» de réhabilitations de quartiers avec mutation des populations, aient été permises dans la mesure où des familles alicantines bourgeoises y trouvaient un fort intérêt. La rénovation et l'aménagement du quai nord et du casq antic ont satisfait des responsables d'entreprises portuaires qui périclitaient depuis les années soixante. Quant à l'implantation de la centralité commerciale maghrébine dans la vieille ville «basse », elle a été accompagnée par les milieux Pied noirs, qui, depuis les années soixante, développaient les circulations maritimes méditerranéennes et avaient acquis, dans la dernière période franquiste, une influence importante sur la bourgeoisie locale.

Les Pieds noirs furent probablement, durant la période franquiste, la première migration qui tenta un renouveau des activités portuaires. Plus de 30000 d'entre eux passèrent par Alicante ou y demeurèrent (12 000). Il s'agissait d'un "retour " de populations expatriées au début du xix ${ }^{\text {ème }}$ siècle : le plus souvent ces personnes avaient perdu tout contact avec leurs origines espagnoles mais, accrochées à l'idée d'une "Algérie française", l'exil dans cette région espagnole si proche d'Oran, autour de leaders de l'OAS « civile », comme Ortiz, leur paraissait être une solution d'attente plus sensée qu'une installation en France. La municipalité et l'État leur concédèrent une frange du domaine public de bord de mer, après la plage du Postiguet, sous le château Maure, au nord du port. Ils construisirent alors un grand ensemble de logements d'architecture verticale et plusieurs milliers d'entre eux habitèrent là, sur la mer. Les 
Pieds noirs n'avaient pas attendu longtemps pour investir dans les activités portuaires : certains d'entre eux utilisèrent leur expérience de gestion de compagnies de navigation internationale en Algérie pour investir dans le cabotage méditerranéen et dans le transport maritime de personnes et de charges moyennes. De nombreux Juifs figuraient parmi eux, qui prirent parole avec des Juifs marocains récemment installés dans la ville. Dès que les Marocains apparurent à Crévillente, dans les années quatre-vingt-dix, et que les Algériens de France se détournèrent, simultanément, de Marseille pour Alicante afin de rejoindre Oran, et même Alger, ces entrepreneurs pieds noirs aperçurent une opportunité pour relancer la navigation de personnes vers le Maghreb. La ligne Alicante Oran fut inaugurée. C'est alors que des locaux furent achetés dans la ville basse et cédés à des Algériens et à des Marocains pour leurs activités commerciales de chargement de véhicules individuels des «fourmis». Ainsi, les Pieds noirs apparaissent en renforcement de cette population que nous avons précédemment signalée: une population voici peu étrangère en renforce une autre actuellement étrangère, sans faire de lien avec d'autres populations d'étrangers. Une ritournelle de l'altérité marque les emprises urbaines de bord de mer, et les tourne vers des contrées lointaines.

\section{Le quartier de l'Institut Européen de Normalisation}

La Communauté européenne a décidé en en 1998 de doter Alicante de l'Institut Européen de Normalisation. Cet organisme fort, à terme, de plus de deux mille salariés dont une moitié d'ingénieurs et cadres supérieurs a été implanté au sud du port, sur la route de Santa Pola et de l'aéroport. Un programme immobilier luxueux fut développé à proximité immédiate des bâtiments de cet organisme. Mais, là, il semble bien que cette nouvelle population déroge des processus précédemment signalés d'installation à Alicante. La diversité d'origine des nouveaux venus ne se prêtait pas à une telle procédure, et les familles firent préférentiellement leur choix résidentiel dans l'ensemble sud et ouest de la conurbation, produisant une sorte d'irisation dans divers quartiers centraux d'Alicante, d'Elche et de la côte vers Santa Pola. Une famille française, homme ingénieur, femme traductrice, deux enfants de 7 et 10 ans, qui nous reçut dans son domicile à l'entrée de Santa Pola expliqua simplement son choix : les plages de sable propre et à quelques minutes de marche, au contraire de celle du Postiguet difficile d'accès à Alicante, l'école proche de l'Institut de Normalisation, où l'on se rend tous les jours pour travailler. L'aéroport voisin, des routes et autoroutes permettant des accès rapides à Elche au Nord-Ouest et Murcia au Sud, des loyers inférieurs de $30 \%$ environ de ceux pratiqués dans les quartiers bourgeois d'Alicante. Autant d'avantages qui semblent augurer, dans les années à venir, du développement d'un pôle international dans la conurbation Sud d'Alicante, et au-delà. Dès lors l'ensemble même des côtes maritimes de la conurbation connaîtra le sort de celles d'Alicante.

\section{La « ville espagnole»}

Pour la majeure partie de la population alicantine, formant la « ville espagnole », qui se réclame rarement de "familles originelles ", ou catalanes (nous sommes à l'extrême sud de la zone d'influence historique, politique et linguistique catalane), et plus souvent d'origines madrilène ou albacetine, les quartiers et populations dont nous venons de parler sont lointains: "gens du Port» résume cette estranéité. Les jeunes 
pratiquent quelques boîtes de nuit du quai nord, mais préfèrent aller vers celles de Santa Pola, dont l'accès est d'un moindre coût. Par contre les grands marchés publics, couverts ou de rue, les nombreux petits commerces, les artisans, mais encore les médecins, avocats et autres professions libérales, sont situés dans cette zone urbaine, désignée comme la "ville espagnole », construite pour partie dans les années vingt, et pour partie dans les années cinquante, à l'ouest du casq antic et de la ville vieille basse. Près du port industriel, au sud, des séries de logements sociaux, en habitat collectif de trois à cinq étages sans espaces verts, exposent des façades fanées. C'est là que résident les ouvriers et employés du port industriel, mais aussi de nombreux petits employés des commerces et entreprises de la « ville espagnole».

Les valeurs du foncier ne semblent pas influencées par la gentrification des quartiers d'étrangers. Le prix moyen $\mathrm{du} \mathrm{m}^{2}$ est de 1400 euros ce que l'on trouve dans les quartiers équivalents de villes de grande homogénéité comme Murcia ou Valencia. La population de la «ville espagnole » a sérieusement baissé, ces cinq dernières années, passant de 205000 à 197000 habitants. Cela contribue au maintien des prix moyens du foncier locatif ou en accession, et à un mouvement d'initiative privée d'agrandissement des surfaces résidentielles, car les « étrangers riches ou pauvres » des quartiers du port ne sont pas intéressés par les offres locales.

La "tournée exotique », comme disent les habitants de la «ville espagnole », se fait en voiture, avec quelques haltes: on commence au sud par le spectacle de l'Institut Européen de Normalisation, puis on remonte par la vieille ville basse, où une halte permet d'acheter quelques bijoux à bas prix et de déjeuner ou de dîner de plats étrangers bon marchés, avant une promenade sur l'Explanada de España, puis de se garer sur le quai nord, pour admirer les yachts et autres bateaux luxueux, de tenter une promenade dans les rues de la "ville anglaise », ex casq antic, et enfin d'observer encore une fois, du surplomb qu'offre la corniche, le quartier pied noir. C'est la "tournée » d'une après-midi entière, pratiquée hebdomadairement par des milliers d'Alicantins, en dehors de la période des bains de mer, au sud du port, dans la direction de Santa Pola, en évitant la plage immédiatement au-dessous de l'Institut de Normalisation. Des «clubs », classiques maisons de passe, se sont installés là avant l'arrivée du personnel européen.

La réciproque à ces comportements n'existe pas : on ne va pas des quartiers d'étrangers dans la ville "espagnole", sinon pour un usage très fonctionnel de telle ou telle boutique ou du grand marché couvert.

\section{Gérer le « cosmopolitisme circulant »}

39 Les autorités municipales, comme leurs opposants politiques, développent une théorie concordante de la gestion urbaine. Les étrangers proches, ou les Maghrébins, et lointains, ou les populations européennes et au-delà, doivent vivre en bonne intelligence avec les alicantins : les uns, étrangers, circulent, déménagent fréquemment et exigent la satisfaction de besoins d'un standard très élevé ou très différent de celui des autochtones, dès lors la séparation physique est nécessaire, et les autorités municipales doivent veiller à permettre l'accès des zones d'étrangers, en aménageant des espaces piétons, des parcs, les quais, des parkings, bref, tout ce qui doit contribuer au plaisir de cette fameuse «tournée " des habitants de la "ville espagnole ». À la question d'une éventuelle construction d'un rapport de voyeurisme en réponse à deux 
types de ségrégations : le premier confinant les Arabes dans un espace spécialisé, la ville basse, avec une ligne de fuite vers la mer et l'autoroute, et la mission de concentrer toutes les pauvretés de la ville, le second ségrégant toutes les populations autochtones de la ville espagnole (« on peut voir le quai nord, mais sans y toucher, c'est comme au zoo, quoiqu'on ne nous fasse pas encore payer l'entrée » nous disait un jeune alicantin de la "ville espagnole») les réponses suggérèrent une politique du développement urbain. En premier lieu, nous dit l'adjoint à l'urbanisme, encadrer les étrangers riches ou pauvres de leurs commerces dans leur propre espace c'est s'assurer la paix civile. En second lieu, installer ces populations remuantes le long du port, de la mer, éloigne de la ville les bruits qu'elles ne manquent pas de faire à toute heure, le plan de circulation permettant l'accès et le dégagement de ces lieux sans halte dans la "ville espagnole ", où d'autres équipements, scolaires, sportifs, culturels, sans intérêt véritable pour les étrangers sont développés. Enfin, la chance que possède Alicante d'une telle articulation au monde ne peut plus se matérialiser, comme par le passé, par quelques maisons noyées au milieu de celles des autochtones, mais bien par des "quartiers monde». Après avoir conjugué, de nombreuses façons différentes, les notions de "développement durable " de "gouvernance " et de "mixité sociale", notre interlocuteur nous affirma que la "mixité sociale existe ici plus qu'ailleurs, mais à une échelle autre " et que la "mission des autorités publiques est d'investir des fonds publics dans les services pour les populations espagnoles, de veiller au bon usage des fonds privés pour et par les populations étrangères, et d'aménager au mieux les circulations ».

\section{Des « districts moraux » transnationaux}

Nos enquêtes ont permis d'identifier ces deux villes comme modèles relativement uniques, les plus affirmés dirons-nous, de la transformation urbaine à partir des mouvements migratoires récents dans le pourtour euro-méditerranéen. Il est à noter que ces deux villes sont dans des positions «frontières » dans les logiques originales de spatialisation dans les territoires circulatoires: elles bornent de vastes espaces criminogènes que les réseaux de migrants commerçants transnationaux évitent ou traversent sans halte, sans exercice de leurs compétences commerciales. Pour Alicante il s'agit de l'Andalousie, région qui concentre les trafics de personnes - sans papiers pour les cultures sous serre, femmes pour la prostitution -, les cigarettes et les psychotropes, le troc de divers produits européens vendus sur les marchés de Casablanca, contre du cannabis à destination d'Allemagne et des Pays-Bas, etc. et pour Sofia de la vaste région "sans État» qui fédère le nord de la Macédoine, l'ouest du Kosovo et le sud du Monténégro, ce que certains désignent comme partie de la "grande Albanie ${ }^{16}$ ». Là, dans une absence totale de services (école, téléphone, transports, poste, électricité,...) des propriétés agricoles se consacrant surtout à l'élevage ovin, hébergent des milliers de "vaincus » des guerres caucasiennes, moyen-orientales et balkaniques, en attente d'une éventuelle reprise d'hostilités autour de l'identité albanaise ou/et musulmane. Les migrants Afghans ou caucasiens qui desservent les Syriens de Sofia ne viennent pas par là, et les psychotropes en provenance d'Afghanistan, qui franchissent massivement ces lieux hautement criminogènes vers l'Italie sont acheminés, passés, par les sociétés locales. C'est ainsi que dans ces zones les frontières entre modèles ailleurs dominants et cloisonnés, voire antagoniques, des économies souterraines d'une part de portée internationale, économies de produits d'usages licites ou de produits d'usages 
illicites, d'autre part de portée locale, deviennent floues, poreuses, et permettent à des individus en circulation de passer de l'un aux autres ${ }^{17}$.

Dans les villes de Sofia et d'Alicante, l'être d'ici et de là-bas supplante, pour les étrangers connectés aux circulants transnationaux, celui, ni d'ici ni de là-bas, décrit par Stonequist, puis, bien plus tard, par Abdelmalek Sayad. Les quartiers les plus centraux, les plus actifs en matière de commerce et de rénovation prennent place désormais dans ces deux villes loin des traditionnels quartiers de relégation, périphéries pauvres ou centres en déshérence, qui accueillent habituellement les migrants assignés à sédentarité.

En réalité tout se passe comme s'il y avait interaction suffisante entre ces villes et les proches zones criminogènes, à la façon, mais hors échelle, dont les " districts moraux » pouvaient, aux Etats-Unis, dans la première moitié $d u x^{\text {ème }}$ siècle, travailler Chicago. La dialectique des transformations est bien en acte, suivant le schéma général simmélien, mais les échelles territoriales sont autres.

Par ailleurs, le débat sur l'étanchéité des portes européennes frontalières turques et marocaines apparaît comme dépassé, désuet, exprimant des visions idéologiques en décalage avec la réalité des circulations ${ }^{18}$. Par contre celui sur la "remontée des Sud" et sur l'inévitable porosité des frontières européennes est bien d'actualité...

\section{BIBLIOGRAPHIE}

DE TAPIA Stéphane (2005) Ulus et Yurt, entre espace nomade et mondialisation. Territoires, espaces et sociétés de l'aire altaïque au champ migratoire turc, Habilitation à Diriger des Recherches, Université de Poitiers, 3 vol. (pag. mult.).

DE TAPIA Stéphane, MULLER Laurent (2005) La création d'entreprises par les immigrés, un dynamisme venu d'ailleurs, Paris,L'Harmattan, $270 \mathrm{p}$.

GAISSAD Laurent (2006) Sexualités cachées : lieux de rencontres homosexuelles dans le sud de la France, Thèse de sociologie, université de Toulouse le Mirail.

MA MUNG Emmanuel (2005) La diaspora chinoise et la création d'entreprises : réseaux migratoires et réseaux économiques en Europe du Sud, in De Tapia Stéphane et Muller Laurent, La création d'entreprises par les immigrés, un dynamisme venu d'ailleurs, Paris,L'Harmattan, pp. 91-108. MISSAOUI Lamia, TARRIUS Alain (1995) Arabes de France dans l'économie souterraine mondiale. L'Aube, 220 p.

MISSAOUI Lamia, TARRIUS Alain (à paraître en 2006) Une Europe sans frontière : la remontée des Sud. PARK Robert E., STONEQUIST Everett V. (1928) The marginal man, Chicago, Chicago University Press.

REMY Jean éd. (1995) Georg Simmel, ville et modernité, Paris, L'Harmattan, 216 p.

SEMPERE SOUVANNAVONG Jean-David (2001) Les pieds-noirs à Alicante, Revue Européenne des migrations internationales, vol. 17, $\mathrm{n}^{\circ} 3$, pp. 173-198. 
TARRIUS Alain (1992) Les Fourmis d'Europe Paris, L'Harmattan, 208 p.

TARRIUS Alain (2001) Les nouveaux cosmopolitismes. L'Aube, 267 p.

TARRIUS Alain (2002) La mondialisation par le bas, Paris, Balland, 169 p.

WELZER-LANG Daniel (2005) La planète échangiste. Payot France, 2005, 214 p.

ZNANIEVSKI Florian, THOMAS William (1999) Le paysan Polonais, Nathan Essais, 1999, 325 p., préface de Pierre Tripier.

\section{NOTES}

1. En premier lieu Thomas et Znanievski, Park, Burgess, Wirth, Halbwachs, Elias, Chombart de Lowe, Goffman. À partir des années quatre-vingt, Yves Grafmeyer et Isaac Joseph relancèrent fortement cette perspective en créant l'option de DEA « cultures urbaines » à l'Université de Lyon 2. Nous avons co-dirigé cette formation, de 1991 à 1993, avec Jean Métral, Alain Battegay et Isaac Joseph.

2. On peut bien sûr considérer que cette position caractérise le parcours du "Paysan Polonais " (Znanievski et Thomas, 1999). Elle est particulièrement exprimée par Park et Stonequist (1928).

3. -Du subi au choisi selon le cheminement du migrant et non selon les dispositions des autorités, comme c'est aujourd'hui le cas en France.

4. C'est ainsi que R. E. Park insistera, dans ses derniers travaux, sur les circulations de l'argent, et plus particulièrement sur les rôles de la banque, des promoteurs immobiliers, etc., dans ces inversions des valeurs morales que représentent les mutations de la valeur monétaire par le blanchiment.

5. Traduction de «moral area » adoptée par Yves Grafmeyer et Isaac Joseph, qui eut peut-être gagné à s'énoncer sous la forme « zone de moeurs".

6. Les théories marxistes de la mobilisation internationale de la force de travail en des lieux précis de la concentration des mains-d'œuvre bon marché ont clairement analysé ce processus «classique » d'appel aux flux migratoires. Désormais l'État français projette de ne mobiliser «que » les élites étrangères, aggravant, si cela est encore possible, le pillage des ressources des États dépendants.

7. Lamia Missaoui et Alain Tarrius ont mené en 2004 et 2005, avec l'aide du Plan Urbanisme, Construction et Architecture (PUCA) des recherches en Europe du Sud sur la « gentrification » de villes particulièrement concernées par les présences de migrants transnationaux : Marocains en Espagne et dans le Sud de la France, Afghans, Pakistanais, Caucasiens, Syriens, dans les Balkans.

8. Enquête menée par Alain Tarrius. Elle a bénéficié de l'aide de notre collègue de l'Académie des Sciences Bulgare, Svetla Koleva, sociologue à l'Académie des Sciences Sociales Bulgare. Le coauteur de l'article utilisera donc le « je » dans cette partie du texte. Pour le reste le «nous » est de rigueur.

9. Cette enquête, dont je ne signalerai que les résultats concernant cet article, entamée grâce à l'aide de chercheurs de l'IFEA d'Istanbul (Institut d'Etudes Anatoliennes d'Istanbul - CNRS et MAE) fut suffisante pour la problématique du changement urbain à Sofia, mais elle peut être considérée comme préalable à une recherche sur les migrations balkaniques.

10. R. Soultan, thèse de doctorat en cours à Bruxelles, sous la direction d'Andréa Réa.

11. Les travaux de notre collègue F. Fontaine, CRH-EHESS, CNRS, sur les colporteurs sont au cœur de cette thématique.

12. Les statistiques sont imprécises : le recensement de 1992 signale 5438 « Arabes » en Bulgarie, comptés comme partie des «populations musulmanes » regroupant $14 \%$ de la population (avec 
les turcophones bulgares et les Pomaks - Slaves islamisés -). Celui de 2001 signale 3000 Syriens, tous statuts confondus, ayant créé plus de 1000 entreprises (commerces, bazars, artisanat) à Sofia... Et une enquête de l'OIM de 1994 signale, elle, 1780 Syriens, 390 Irakiens, 275 Iraniens et 129 Afghans possédant un statut de résident permanent ou de longue durée. Les données les plus récentes (2004) et les plus fiables sont fournies par l'ONG de Sofia Icmsi qui compte 34000 «Arabes » (définition recensement de 1992). Les présences de Pomaks ayant peu varié et celles de turcophones plutôt baissé (crise de 1996), les présences d'«Arabes de l'extérieur » ont donc fortement augmenté.

13. Dans mon enquête à Dragalevtsi j'ai été aidé par S. T., une Bulgare de quarante cinq ans, fille d'ambassadeur, études secondaires et supérieures en France et en Suisse, un doctorat de chimie obtenu en Bulgarie. La disgrâce de son père, en 1991 l'a contrainte à vendre des sandwiches dans une petite échoppe d'une place de Dragalevtsi où sa famille essaie de conserver une maison acquise dans les années quatre-vingt (en louant aussi deux niveaux).

14. Là comme ailleurs, le Rom est stigmatisé et érigé en arbre qui cache la forêt. Nous avons de nombreux contre-témoignages, qui marginalisent le rôle des Roms dans ces trafics. Le grand nombre d'entre eux arrêtés pour de minuscules implications suffit à remplir les prétoires et donc, probablement, à faciliter les trafics à l'initiative d'autres populations.

15. Nos enquêtes (Lamia Missaoui et Alain Tarrius) à Alicante se sont déroulées en 2000 (avec l'aide de Juan David Sempere, professeur à l'Université d'Alicante), puis en 2002, et enfin en 2005.

16. En fait les régions albanophones s'étendent jusqu'en Roumanie.

17. Nous menons actuellement une recherche "à compte d'auteur " sur ces phénomènes de permutations, chevauchements, des logiques migratoires et des références normatives qui les identifient, le long d'une "grande transversale ", de Turquie et du Caucase jusqu'au Portugal (Tarrius et Missaoui, à paraître en 2006).

18. Nous n'avons pas ici abordé les perspectives ouvertes par les circulations des populations turques. Les analyses de Stéphane de Tapia confirment les points de vue exposés dans cet article. Voir de Tapia et Muller, 2005 ; de Tapia, 2005. Les analyses d'Emmanuel Ma Mung sur la diaspora commerçante chinoise, et, particulièrement, le rapport entre Paris et l'Italie contribuent aussi à « délocaliser » le regard des frontières de la ville vers de vastes espaces transnationaux. Voir Ma Mung, 2005.

\section{RÉSUMÉS}

Les auteurs étudient depuis une vingtaine d'années les déplacements internationaux de migrants petits entrepreneurs commerciaux. L'intensification et la densification de ces activités et de ces populations les ont amenés à identifier une nouvelle forme migratoire typologiquement différente des formes diasporiques. Leurs approches compréhensives et interactionnistes de populations en réseaux, liées par la parole, l'honneur, qui excèdent des frontières et des normes sociales des divers États-nations qu'elles traversent ou habitent, leur ont permis de proposer la notion de «territoires circulatoires": il s'agit d'une topique spatio-temporelle concernant l'ensemble des transactions et interactions, des rapports symboliques et matériels exprimant ces mobilisations internationales, et susceptibles de produire ou d'héberger des rapports sociaux originaux.

Cette approche d'une sorte de territoire de la mondialisation «par le bas » transversal aux espaces des nations, présente l'intérêt de maintenir l'attention des chercheurs sur des faits 
d'interactions fugitifs, marginaux, masqués, mais très révélateurs, que les approches plus systémiques (logistiques, flux, etc..) ont tendance à gommer. Toutefois, les auteurs se défendent de dissocier topiques des circulants et des sédentaires, faits exposés et faits masqués, officialité et subterranéité. Dans cet article, ils montrent que les échelles spatio-temporelles caractéristiques des territoires des circulants s'articulent à celles des sédentaires dans des villes proches des «frontières- clefs » de l'Europe : c'est ainsi qu'ils décrivent comment migrants circulants et sédentaires contribuent aux transformations urbaines de Sofia, en Bulgarie et d'Alicante, en Espagne. Ils revisitent alors les propositions de Robert Ezra Park, et d'autres chercheurs héritiers de la sociologie de Georg Simmel, en matière de processus de transformations urbaines, montrent l'intérêt et l'actualité de leurs analyses, mais aussi proposent, à partir de leurs travaux, des changements d'échelles spatiales et temporelles appelées dans ces théories.

Towns and migrants : from world as a place ('lieu-monde') to world as a thouroughfare ('lieu-passage'). The authors have been studying for some 20 years international movements among small tradespeople. As these became more numerous and more intricate, those people and their movements brought the authors to outline/ define a new form of migration, typically different from the diaspora type migrations. A comprehensive and interactionist approach of people, defined as networks, bound by word given and honour, stretching beyond the boundaries and social norms of the various nation-states they pass through or live in, allowed the authors to propose the notion of 'circulation territories' (territoires circulatoires). It is a spatio-temporal topic, which covers all transactions and interactions, all symbolic and concrete relations, which express those international forms of mobilization, and which are likely to produce or harbour new types of social relations.

Delineating some kind of space for an 'upward' globalisation, across national territories, offers the opportunity to keep researchers aware of transient, marginal, hidden interactions, which are very significant, but which more systemic approaches (in terms of logistics, of flow, etc) tend to erase / blur. However, the authors do not separate obvious and hidden facts, official and subterranean acting, schemes relating to those who move and those who stay. In the present paper, they show that the spatio-temporal scales, which are typical of the territories trodden by those who move, link up with those of sedentary migrants in towns close to 'key boundaries' in Europe. They show how 'circulating' and 'sedentary' migrants contribute to urban change in Sofia, Bulgaria, and Alicante, Spain. In so doing, the authors revisit the proposals made by Robert Ezra Park and other researchers, followers of Georg Simmel's sociology regarding the processes of urban change, they point out at the importance and topicality of these approaches. But, on the basis of their own work, they also propose a change in the spatial and temporal scale of their theories.

Ciudades e inmigrantes, del lugar-mundo al lugar-pasaje. Desde hace veinte años, los autores estudian los desplazamientos internacionales de inmigrantes pequeños comerciantes. La intensificación y la densificación de estas actividades y de estas poblaciones han conducido los autores a identificar una forma migratoria nueva de tipo diferente del de las formas de diásporas. Sus visiones comprehensivas e interaccionistas de poblaciones en red, ligadas por la palabra y el honor y que exceden fronteras y normas sociales de los diversos Estados-naciones que atraviesan o habitan, han permitido a los autores proponer la noción de «territorios circulatorios »: se trata de una característica espacio-temporal que concierne el conjunto de las transacciones e interacciones, de las relaciones simbólicas y materiales que exprimen estas movilizaciones internacionales susceptibles de producir o albergar relaciones sociales originales.

Esta visión de una especie de territorio de la mundialización "por abajo ", transversal a los espacios de las naciones, presenta el interés de mantener la atención de los investigadores en hechos de interacciones fugitivos, marginales, escondidos pero muy reveladores y que las visiones más sistémicas (logísticas, flujos, etc.) tienden a alisar. Con todo, los autores no disocian 
caracteres de circulantes y sedentarios, hechos expuestos y escondidos, oficialidad y «subterraneidad ». En este artículo, muestran que las escalas espacio-temporales características de los territorios de aquellos que circulan se articulan con las de los sedentarios en las ciudades próximas de las «fronteras-clave" de Europa: describen así cómo inmigrantes circulantes y sedentarios contribuyen a las transformaciones urbanas de Sofía en Bulgaria y de Alicante en España. Reanalizan las proposiciones de Robert Ezra Park y de otros investigadores herederos de la sociología de Georg Simmel en materia de procesos de transformaciones urbanas y muestran el interés y la actualidad de sus análisis proponiendo también, a partir de sus propios trabajos, cambios de escalas espaciales y temporales en estas teorías.

\section{INDEX}

Mots-clés : entrepreneurs, territoire, mobilité géographique

\section{AUTEURS}

\section{LAMIA MISSAOUI}

Maître de conférences de sociologie, Université de Versailles Saint Quentin en Yvelines, 47 bd Vauban, 78047 Guyancourt, France. Courriel : lamia.missaoui@libertysurf.fr

\section{ALAIN TARRIUS}

Professeur de sociologie et d'anthropologie urbaine, Université de Toulouse le Mirail, 5 Allée Antonio Machado, 31058 Toulouse cedex 9, France. Courriel : Altarrius@aol.com 